\title{
Impact of Nodal Staging for Small Bowel Carcinoid: More is Better!
}

\author{
Thuy Tran, Heidi Reich, Anna Leung, Edward Wolin, Farin Amersi, Danielle M Hari
}

\begin{abstract}
Current guidelines for small bowel carcinoid (SBC) do not specify the extent lymph node (LN) staging required. Our aim was to determine whether analyzing more LNs in patients who undergo surgical resection for SBC correlated with improved survival. The Surveillance, Epidemiology, and End Results (SEER) database was queried to identify patients undergoing surgical resection for histologically confirmed SBC from 19882009. The number of $L N$ s examined was categorized into 0,1 to 6,7 to 11 and $12+$ nodes. Patient demographics and tumor characteristics were assessed by Logrank and Cox regression analysis. The median number of LNs examined for all 2796 patients was 4.00 (mean 6.83). For all stages of jejunal and ileal tumors examined, increased nodal sampling was associated with significantly improved survival $(p<0.001)$. On regression analysis, older age, non-white race, larger tumor size, higher T-stage and number of LNs examined were predictive of worse survival. For jejunal and ileal SBC, adequate lymphadenectomy, consisting of 12 lymph nodes, correlates with significantly improved survival. Presently, no guideline exists for SBC LN staging, but it is critical to ensure adequate surgical staging in patients who undergo resection for jejunal and ileal SBC.
\end{abstract}

Keywords: Small bowel carcinoid, Lymph nodes, Survival.

How to cite this article: Tran $T$, Reich $H$, Leung A, Wolin $E$, Amersi F, Hari DM. Impact of Nodal Staging for Small Bowel Carcinoid: More is Better! World J Endoc Surg 2013;5(3):65-70.

Source of support Nil

Conflict of interest: None

\section{INTRODUCTION}

Carcinomas of the small intestine account for less than 5\% of all gastrointestinal (GI) malignancies, yet recent studies have demonstrated a worldwide increase in the number of neuroendocrine tumors of the small intestine. ${ }^{1}$ In the past four decades, there has been a 300 to $500 \%$ increase in the incidence of neuroendocrine tumors in the $U$ nited States as well as Sweden and N orway. ${ }^{2-6} \mathrm{~N}$ early $75 \%$ of all carcinoids are found in the $\mathrm{GI}$ tract with the small bowel being the most common location (45\%). ${ }^{6,7}$

Small bowel carcinoids (SBC) typically present with metastatic disease, but several studies have demonstrated long-term overall survival for all stages. The current $N$ ational Comprehensive Cancer Network (NCCN) guidelines for locoregional SBC tumors include specific options for management based on primary tumor site. ${ }^{8} \mathrm{M}$ ore specifically, duodenal SBC tumors can be treated with endoscopic resection or local surgical resection. Lymphadenectomy $(L A D)$ is not required for duodenal tumors while bowel resection with regional $L A D$ is required for jejunal and ileal tumors. However, there are currently no specified guidelines regarding the extent of $L N$ examination for SBC.

U sing a population-based study, we wanted to determine whether analyzing more lymph nodes ( $\mathrm{LN} \mathrm{s}$ ) in patients who undergo surgical resection for SBC improves survival.

\section{MATERIALS AND METHODS}

W econducted a population-based retrospective study between 1988 and 2009 using the Surveillance, Epidemiology and End Results (SEER) database, which is one of the largest cancer research sources from the $\mathrm{N}$ ational $\mathrm{C}$ ancer Institute. The SEER database consists of 13 population-based cancer registries that represent approximately $28 \%$ of cancer patients in the U nited States. The data is collected from cancer registries across the United States. It includes information such as demographic, primary site, geographic region, histology, stage at diagnosis, treatment and tumor characteristics. We queried the database for patients with a histologic diagnosis of carcinoid involving the following sites: duodenum, jejunum, ileum and other overlapping lesions. Malignant carcinoids of the small intestine were defined using SEER codes for cancer site and histology. Tumor characteristics were analyzed, including tumor location, tumor size, tumor grade and AJCC TNM stage. We excluded all patients with distant metastatic disease, unknown number of $\mathrm{LNS}$ examined and more than one primary malignancy. Univariate survival analysis was performed using Kaplan M eier method and log-rank test. Multivariate survival analysis was performed using Cox hazard proportional model adjusting for age, gender, race, year of diagnosis, tumor location, tumor size, tumor grade, stage, number of and lymph nodes examined. A ll statistical analysis was conducted using SPSS version 19.0 software (Chicago, IL, USA), with $p$-value less than 0.05 considered significant.

\section{RESULTS}

Of the 11,510 patients identified, 7,078 were excluded for inadequate staging information, 776 were excluded for metastatic disease and 860 were excluded for having more than one primary. After exclusion criteria were applied, 
2,796 patients were eligiblefor analysis. Patient demographics based on the number of $L N S$ examined are shown in Table 1. Kaplan-M eier survival curves demonstrated a survival benefit for patients with at least $1 \mathrm{LN}$ examined compared to those with $0 \mathrm{LNs}$ examined (Graph $1 \mathrm{~A}$, $p<0.001$ ). For duodenal SBC, there was no statistically significant difference in survival based on whether $L N$ s were examined (Graph 1B, $p=0.827$ ). However, for jejunal and ileal SBC, examining at least $1 \mathrm{LN}$ correl ated with a survival benefit compared to those with $0 \mathrm{LN}$ s examined (G raph $1 \mathrm{C}$, $p<0.001$ ). A dditionally, for jejunal and ileal SBC, KaplanM eier survival curves demonstrated statistically significant improvement in overall survival in those with $>12 \mathrm{LN} \mathrm{s}$ examined compared to those with 0, 1-6 and 7-11 LN S (Graph 2, $p<0.001$ ). This improvement in survival was maintained after a subgroup analysis based on A J CC staging for jejunal and ileal SBC was performed for node negative (stage I/II/IIIa, Graph 3A, $p=0.003$ ) and node positive (stage IIIb, Graph 3B, $p=0.001$ ) patients.

A fter adjusting for patient demographics, tumor characteristics and TNM stage, multivariable regression analysis demonstrated that examination of less than $12 \mathrm{LN} \mathrm{S}$ correlated with a decrease in survival for with jejunal and ileal SBC. Other factors that positively affected survival included younger patient age, male gender, white race, tumor size less than $1 \mathrm{~cm}$ and lower T-stage (Table 2).

\section{DISCUSSION}

Historically, the management of GI carcinoids had been challenging due to insufficient data regarding predictive

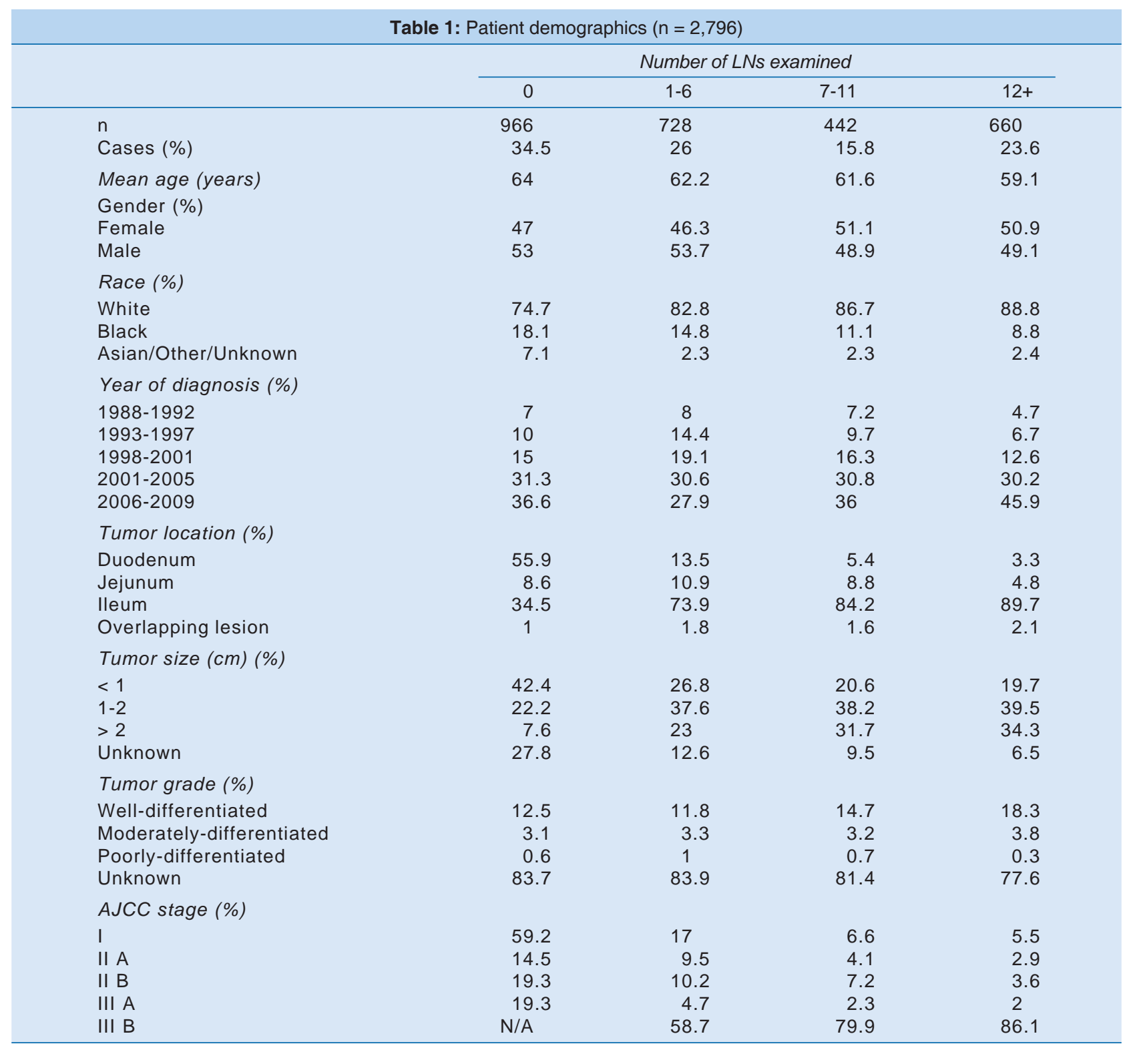


prognostic variables. As a result, a meaningful staging system did not exist. Over time, several studies revealed a significant difference in tumor biology and survival based on the primary organ site of carcinoid tumors. ${ }^{6,9-12} \mathrm{~V}$ arious studies have reported a superior 5-year observed survival rates for localized carcinoids of the rectum $(90 \%)$, appendix (88\%) and colon (85\%) when compared to the cecum (68\%)
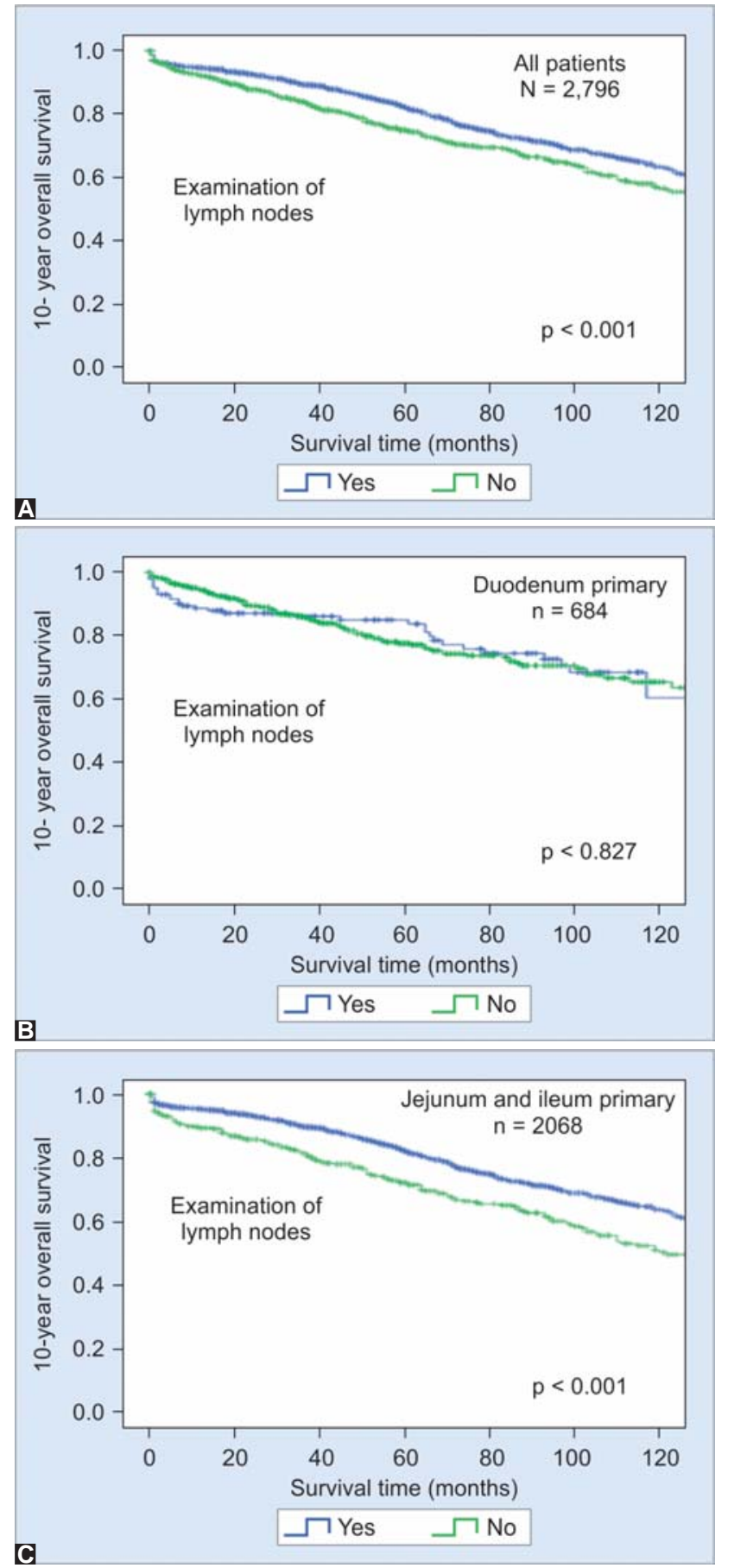

Graphs 1A to C: Overall survival based on whether lymph node were examined (A) All patients, (B) Duodenal primary, (C) Jejunal and ileal primary. Yes $=$ at least 1 lymph node examined and $\mathrm{No}=$ 0 lymph nodes examined and small bowel (65-73\%). ${ }^{13}$ These discrepancies led to the establishment of a separate staging system for $\mathrm{GI}$ carcinoids based on the primary organ site. ${ }^{14}$

$W$ ith the incidence of $S B C$ on the rise in many countries such as the U nited States, Norway and Sweden, SBC are now the most common carcinoid of the GI tract.11,12,15,16 The clinical manifestations of SBC are typically vague and nonspecific. They are commonly found incidentally at the time of laparoscopy or endoscopy. Patients with local and regional disease are thought to have a favorable prognosis, but a majority of patients present with metastatic disease. A dvances in endoscopy, double balloon enteroscopy computed-topography (CT) and magnetic resonance imaging (MRI) have improved our ability to detect and clinically stage SBC. ${ }^{17-22}$

Upper gastrointestinal series (UGIS)/small-bowel follow-up through (SBFT) remain a mainstay in the evaluation of SB tumors due to its simplicity, diagnostic value and cost. The utilization of double contrast method of enteroclysis has increased the detection sensitivity to almost $90 \%$; however, it is only used in 50 to $60 \%$ patients being evaluated for SB pathology due to limitations such as lack of visualization of a luminal lesion and the need for a highly-skilled radiologist. ${ }^{23} \mathrm{CT}$ enteroclysis (CTE) has become a superior modality for the evaluation of SBC because it allows the exploration of the entire $\mathrm{SB}$ as well as extraintestinal involvement. CTE can detect SBC that are only $5 \mathrm{~mm}$ in size with a negative and positive predictive value for SBC of 100 and $94.7 \%$ respectively. ${ }^{24,25}$ Similarly, M R enteroclysis (M RE) has also been advocated due to its ability to assess mucous membranes and pathological changes around the bowel for SBC, and studies have shown a $95 \%$ diagnostic accuracy for SB tumors. ${ }^{20,21,26}$

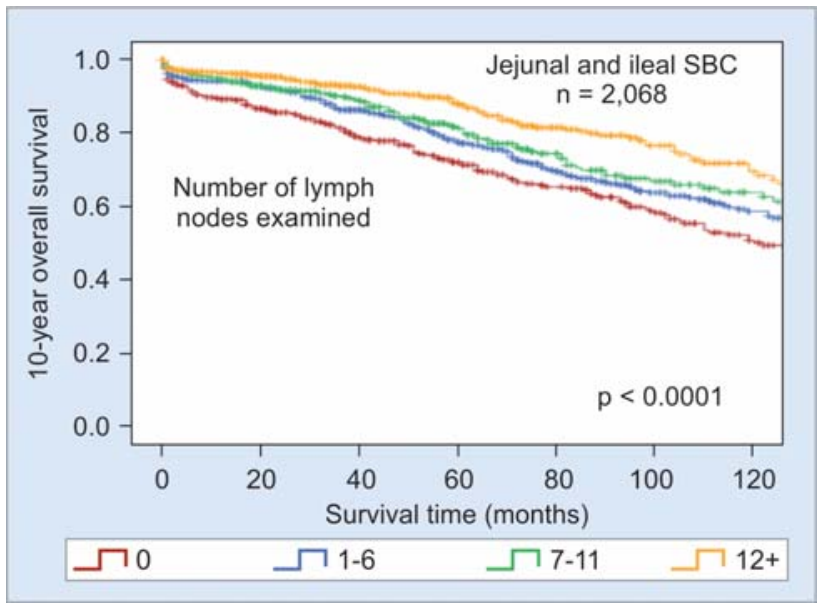

Graph 2: Survival based on the number of lymph nodes examined and for stage I-IIIB patients with jejunal and ileal primary 
M ore than 20 years ago, capsule endoscopy technology emerged as a superior modality for identification of SB tumors and the advancements in camera technology and image capture have revolutionized the technique. ${ }^{22,27}$ However, the advent of push-pull endoscopy (doubleballoon) has challenged the need for capsule endoscopy given its superior diagnostic yield, superior screening ability and therapeutic potential for SBC. Capsule endoscopy and push-pull endoscopy are now advocated as complementary studies in the work-up of SB tumors. ${ }^{23,27}$ A dditionally, for patients with carcinoid syndrome, octreotide scanning is a powerful tool for localizing SBC with a $90 \%$ sensitivity rate, but for those lacking carcinoid symptoms, the sensitivity rate drops to $60 \% .{ }^{19,23}$
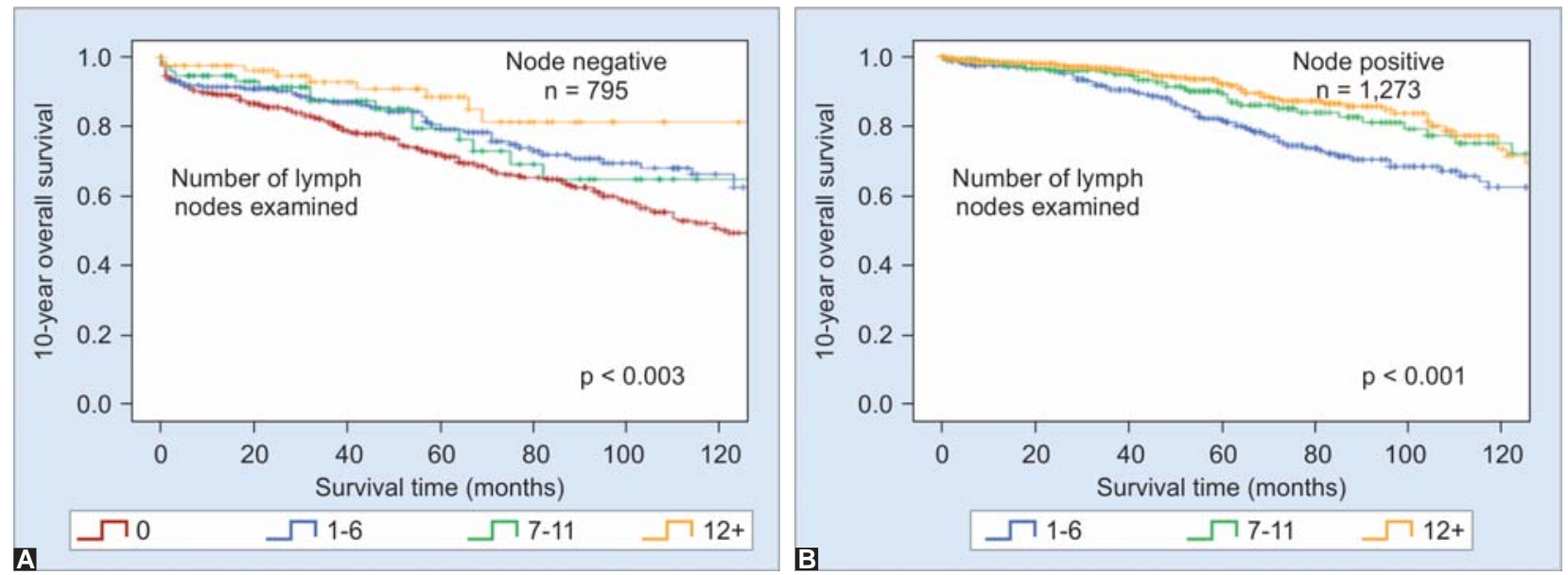

Graphs $3 A$ and B: Survival based on the number of lymph nodes examined and stage for patients with jejunal and ileal primary, (A) AJCC stage I/II/IIIa (node negative), (B) AJCC stage IIIb (node positive)

Table 2: Adjusted Cox proportional hazards regression model for 10-year overall survival in patients with stage I to III small bowel carcinoid for jejunum and ileum tumors

\begin{tabular}{|c|c|c|c|}
\hline Category & Subcategory & Hazard ratio & $\mathrm{p}$-value \\
\hline Age & per year & 1.07 & $<0.001$ \\
\hline \multirow[t]{2}{*}{ Gender } & Male & 1 & 0.025 \\
\hline & Female & 1.202 & \\
\hline \multirow[t]{3}{*}{ Race } & White & 1 & 0.006 \\
\hline & Black & 1.545 & \\
\hline & Asian/O ther/Unknown & 1.229 & \\
\hline \multirow[t]{5}{*}{ Year of diagnosis } & $1988-1992$ & 1 & 0.223 \\
\hline & 1993-1997 & 0.711 & \\
\hline & $2008-2001$ & 0.646 & \\
\hline & $2002-2005$ & 0.673 & \\
\hline & $2006-2009$ & 1.01 & \\
\hline \multirow[t]{3}{*}{ Tumor location } & Ileum & 1 & 0.942 \\
\hline & J ejunum & 0.999 & \\
\hline & Overlapping lesion & 0.873 & \\
\hline \multirow[t]{4}{*}{ Tumor size $(\mathrm{cm})$} & $<1$ & 1 & $<0.001$ \\
\hline & $1-2$ & 0.922 & \\
\hline & $>2$ & 1.228 & \\
\hline & Unknown & 1.443 & \\
\hline \multirow[t]{4}{*}{ Tumor grade } & Well-differentiated & 1 & 0.411 \\
\hline & Moderately-differentiated & 2.016 & \\
\hline & Poorly-differentiated & 1.126 & \\
\hline & Unknown & 1.303 & \\
\hline \multirow[t]{4}{*}{ T-stage } & $\mathrm{T} 1$ & 1 & 0.046 \\
\hline & $\mathrm{T} 2$ & 1.092 & \\
\hline & T3 & 1.334 & \\
\hline & T4 & 1.396 & \\
\hline \multirow[t]{2}{*}{$\mathrm{N}$-stage } & NO & 1 & 0.807 \\
\hline & N1 & 5.723 & \\
\hline \multirow[t]{4}{*}{ Nodes examined } & 0 & 1 & 0.031 \\
\hline & $1-6$ & 0.901 & \\
\hline & $7-11$ & 0.798 & \\
\hline & $12+$ & 0.194 & \\
\hline
\end{tabular}


Several studies have challenged the hypothesis that advances in technology have increased detection of SBC as the explanation to the increased incidence of $S B C$ in the past few decades. ${ }^{5,9,16}$ Furthermore, overall survival has remained constant for all SBC, but there has been conflicting data regarding the prognosis of $S B C$ based on primary tumor site and more importantly, the TNM staging when comparing duodenal and jejunal or ileal SBC. ${ }^{3,4,28}$ The current NCCN guidel ines contain varying surgical treatment algorithms based on primary site of the SBC rather than TNM stage. ${ }^{8}$ J ejunal and ileal tumors were considered to have a poorer prognosis compared to duodenal SBC and other $\mathrm{GI}$ carcinoids. The current standard of care is that duodenal SBC tumors can be treated with endoscopic resection or local surgical resection without lymph node sampling while bowel resection with regional $L A D$ is recommended for jejunal and ileal tumors.

Lymph node involvement remains a significant predictor of survival for $\mathrm{GI}$ adenocarcinomas and carcinoids, yet unlike $\mathrm{GI}$ adenocarcinomas, there are currently no specified guidelines regarding the extent of $L N$ examination for $\mathrm{GI}$ carcinoids. ${ }^{29-31}$ Our study demonstrated that the lack of lymph node examination for all SBC was associated with an inferior 10-year survival compared to patients with at least $1 \mathrm{LN}$ examined (56.5\% vs $63.2 \%$ respectively, $p<0.001$ ). While this was statistically significant for all SBC patients, this correlation was not observed for duodenal SBC (64\% vs $60 \%, p=0.827)$ compared to jejunal/ ileal SBC ( $50 \%$ vs $63.5 \%, p<0.001)$. This supports the $N C C N$ guidelines that $L N$ examination is not necessary for duodenal SBC.

Analysis of the extent of $L N$ examination for jejunal and ileal SBC revealed that patients with at least $12 \mathrm{LN}$ examined had a 10 -year survival of $69 \%$ compared to those with $0,1-6$ or $7-11 \mathrm{LN}$ examined $(50 \%, 58 \%$ and $63 \%$, respectively, $p<0.001)$. A subset analysis of these patients based on $L N$ examination and $L N$ positivity confirmed the importance of adequate $L N$ examination regardless of $L N$ positivity status. Currently, node-negative SBC include Stage I (TINOMO), Stage IIA (T2NOMO), Stage IIB (T3N OM O) and Stage IIIA (T 4N OM O) while node-positive SBC include Stage IIIB (T1-4N 1M 0). ${ }^{14}$ For nodenegative $\mathrm{SBC}$ of the jejunum and ileum, patients with at least 12 L N s examined had a superior 10-year survival advantage compared to those with less or zero ( $72 \%$ vs $63 \%$ and $50 \%$, respectively, $p=0.003$ ). For node-positive SBC of the jejunum and ileum, patients with at least $12 \mathrm{LN}$ s examined had a superior 10-year survival advantage compared to those with $1-6,7-11$ and $>12(62 \%$ vs $72.1 \%$ vs $73.3 \%$, respectively; $p=0.001)$. M ultivariable regression model for SBC of the jejunum ileum confirmed that the number of $L N$ s examined remained a positive predictor of survival.

One reason for the significantly improved survival is likely multifactorial, but the stage migration phenomenon must be considered given that the higher number of $L N$ examined theoretically decreases the risk of missed nodal involvement. However, we observed that both node-positive and node-negative patients with SBC of the jejunum and ileum had significantly improved survival if at least $12 \mathrm{LNS}$ were examined. $V$ ariations in pathologic processing and technique as well as extent of surgical resection must also be considered as an increasing mindfulness of total number of $L N$ s examined. Tumor size and $T$-stage are an integral part of the TNM staging for SBC. In addition to the number of $\mathrm{LN}$ s examined, our study confirmed that smaller, welldifferentiated tumors had a superior survival advantage after multivariable analysis, while nodal involvement was not an independent predictor of survival for jejunal and ileal SBC.

There are several limitations to using a population-based database. The database lacks information on margin status which may be critical for duodenal SBC due to the limited resections done at this site compared to jejunal and ileal tumors. Patient co-morbidities and perioperative complications are also not available in the SEER database. While chemotherapy data is not available in the SEER database, the majority of SBC patients with locoregional disease are treated with excision or surgical resection only so it is unlikely the addition of this data in our study population would impact the survival and conclusions of our study. We also excluded patients with more than one primary and it is common for patients with SBC to have more than one primary. Lastly, recurrence data is not available in the SEER database.

\section{CONCLUSION}

In the largest study to date evaluating the impact of $L N$ analysis in SBC, we demonstrate that the extent of $L N$ examination is important for both node-negative and nodepositive SBC of the jejunum and ileum. Extent of lymphadenectomy correlated with significantly improved survival. Based on our findings, we recommend that surgeons should aim to achieve a minimum of 12 nodes as part of the regional lymph node dissection for SBC of the jejunum and ileum. Presently, no mandate exists for SBC LN staging, but it is critical to ensure adequate surgical staging in patients who undergo resection for jejunal and ileal SBC. Further investigations should be done to confirm these findings. 


\section{REFERENCES}

1. Siegel $R, N$ aishadham $D$, J emal A. Cancer statistics. CA Cancer J Clin 2012;62:10-29.

2. Hauso 0 , Gustafsson BI, Kidd M, et al. N euroendocrine tumor epidemiology: contrasting N orway and N orth A merica. Cancer 2008; 113:2655-2664.

3. Helland SK, Prosch AM, Viste A. Carcinoid tumours in the gastrointestinal tract-a population-based study from W estern N orway. Scand J Surg 2006;95:158-161.

4. Hemminki K, Li X. Incidence trends and risk factors of carcinoid tumors: a nationwide epidemi ologic study from Sweden. Cancer 2001;92:2204-2210.

5. Landerholm K, Zar N, A ndersson RE, et al. Survival and prognostic factors in patients with small bowel carcinoid tumour. Br J Surg 2011;98:1617-1624.

6. $Y$ ao JC, Hassan $M$, Phan $A$, et al. One hundred years after carcinoid: epidemiology of and prognostic factors for neuroendocrine tumors in 35,825 cases in the United States. J Clin Oncol 2008;26:3063-3072.

7. Ghevariya V, M alieckal A, Ghevariya N, etal. Carcinoid tumors of the gastrointestinal tract. South M ed J 2009;102:1032-1040.

8. Kulke M H, Benson A B, 3rd, B ergsland $E$, et al. Neuroendocrine tumors. J N atl Compr Canc Netw 2012;10:724-764.

9. Bilimoria KY, B entrem DJ, W ayne J , et al. Small bowel cancer in the U nited States: changes in epidemiology, treatment, and survival over the last 20 years. A nn Surg 2009;249:63-71.

10. M odlin IM, L ye KD, Kidd M. A 5-decade analysis of 13,715 carcinoid tumors. Cancer 2003;97:934-959.

11. Severson RK, Schenk M, Gurney J G, et al . Increasing incidence of adenocarcinomas and carcinoid tumors of the small intestine in adults. Cancer Epidemiol Biomarkers Prev 1996;5:81-84.

12. Soga J. Early-stage carcinoids of the gastrointestinal tract: an analysis of 1914 reported cases. Cancer 2005;103:1587-1595.

13. http://seer.cancer.gov/statfacts/html. 2012.

14. A JCC Cancer Staging M anual. 7th ed. New Y ork (NY) 2010.

15. Chow JS, Chen CC, A hsan H, Neugut A I. A population-based study of the incidence of malignant small bowel tumours: SEER, 1973-1990. Int J Epidemiol 1996;25:722-728.

16. Scherubl H, Jensen RT, Cadiot G, et al. Neuroendocrine tumors of the small bowels are on the rise: early aspects and management. W orld J Gastrointest Endosc 2010;2:325-334.

17. A nzidei $M, N$ apoli $A$, Zini $C$, et al. M alignant tumours of the small intestine: a review of histopathology, multidetector CT and M RI aspects. Br J Radiol 2011;84:677-690.

18. Capurso $G$, Rinzivillo $M$, Bettini $R$, et al. Systematic review of resection of primary midgut carcinoid tumour in patients with unresectable liver metastases. Br J Surg 2012;99:1480-1486.

19. Koornstra JJ, de V ries EG, Porte RJ . Improvements in small bowel carcinoid diagnosis and staging: 18F-DOPA PET, capsule endoscopy and double balloon enteroscopy. Dig Liver Dis 2009;41:e35-38.

20. Masselli G, Polettini E, Casciani E, et al. Small-bowel neoplasms: prospective evaluation of $M R$ enteroclysis. Radiology 2009;251: 743-750.

21. Pappalardo G, Gualdi G, Nunziale A, et al. The impact of magnetic resonance in the preoperative staging and the surgical planning for treating small bowel neoplasms. Surg Today 2012.

22. Westerhof J, Koornstra JJ, Hoedemaker RA, et al. Diagnostic yield of small bowel capsule endoscopy depends on the small bowel transit time. W orld J Gastroenterol 2012;18:1502-1507.
23. Cheung DY, Choi M G. Current advancein small bowel tumors. Clin Endosc 2011;44:13-21.

24. Hakim FA, Alexander JA, Huprich JE, et al. CT -enterography may identify small bowel tumors not detected by capsule endoscopy: eight years experience at $\mathrm{M}$ ayo Clinic Rochester. Dig Dis Sci 2011;56:2914-2919.

25. K amaoui I, De-Luca V, Ficarelli S, et al. Value of CT enteroclysis in suspected small-bowel carcinoid tumors. AJR A m J Roentgenol 2010;194:629-633.

26. $V$ an Weyenberg SJ, Meijerink $M R$, Jacobs $M A$, et al. $M R$ enteroclysis in the diagnosis of small-bowel neoplasms. Radiology 2010;254:765-773.

27. A dler SN, Bjarnason I. W hat we have learned and what to expect from capsule endoscopy. W orld J Gastrointest Endosc 2012;4: 448-452.

28. Landry CS, Brock G, Scoggins CR, et al. A proposed staging system for small bowel carcinoid tumors based on an analysis of 6,380 patients. A m J Surg 2008;196:896-903 discussion 903.

29. Bilimoria KY, Bentrem DJ, Stewart AK, et al.: Lymph node evaluation as a colon cancer quality measure: a national hospital report card. J Natl Cancer Inst 2008;100:1310-1317.

30. Chen SL, Bilchik AJ . M ore extensive nodal dissection improves survival for stages I to III of colon cancer: a population-based study. A nn Surg 2006;244:602-610.

31. Hellan M, Sun CL, A rtinyan A, et al. The impact of lymph node number on survival in patients with lymph node-negative pancreatic cancer. Pancreas 2008;37:19-24.

\section{ABOUT THE AUTHORS}

\section{Thuy Tran}

Resident, Department of Surgery, Cedars-Sinai M edical Center Los A ngeles, USA

\section{Heidi Reich}

Resident, Department of Surgery, Cedars-Sinai M edical Center Los A ngeles, USA

\section{Anna Leung}

Fellow, Department of Surgical Oncology, John Wayne Cancer Institute, California, USA

\section{Edward Wolin}

Resident, Department of M edicine, Cedars-Sinai Medical Center Los A ngeles, USA

\section{Farin Amersi (Corresponding Author)}

Surgical Oncologist, D epartment of Surgical Oncology, Cedars-Sinai M edical Center, Los A ngeles, USA, e-mail: farin.amersi@ cshs.org

\section{Danielle M Hari}

Surgical O ncologist, Department of Surgical Oncology, J ohn W ayne Cancer Institute, California, USA 